\title{
Muon Detection in the ATLAS CSC Detector
}

\author{
David Primor, Giora Mikenberg, and Hagit Messer, Fellow, IEEE
}

\begin{abstract}
The LHC and its participating experiments create a challenging data processing environment, characterized by a large amount of data in which only a small portion is expected to carry new scientific information. This publication addresses the problem of muon track detection in a Cathode Strip Chamber (CSC), a component of the ATLAS Muon Spectrometer. A new algorithm, based on several novel ideas is introduced. The detect-before-estimate approach is presented, which first detects the muon track using a modified Hough transform, and then estimates the precise hit locations. The muon track detection is improved by taking into account additional, previously unused, information. It is shown that in the presence of high radiation background, the new detection procedure reduces the fake track identification rate significantly. For each track candidate, the hit cluster quality is calculated. It is then shown that including only good quality clusters in the track fitting algorithm, results in a better track parameter estimation. The algorithm is tested with real data taken from test beam, and evaluated using theoretical tools, especially developed for this problem.
\end{abstract}

Index Terms-CSC detector, detect before estimate, mask hit information, signal processing for particle detection, track identification.

\section{INTRODUCTION}

$\mathbf{T}$ HE LHC, the largest hadron collider ever built, presents new challenges for physicists and engineers. With the anticipated luminosity of the LHC, one expects as many as one billion total collisions per second, of which at most 10 to 100 might be of potential scientific interest. The track reconstruction algorithms applied at the LHC will therefore have to reliably reconstruct tracks of interest in the presence of background hits.

One of the two major, general-purpose experiments at LHC is called ATLAS. Since high momentum muons are expected to be among the most important signatures of new phenomena, a stand-alone muon detector system is being built for ATLAS. This system is also called the muon spectrometer [1]. The ATLAS muon spectrometer is located in high radiation background environment which makes the muon tracking a very challenging task. In the inner station of the ATLAS muon spectrometer, Cathode Strip Chambers (CSC) will be employed for the precision measurement of muon tracks in the forward region $(2 .<\eta<2.7$ where $\eta$, the pseudorapidity angle, is defined as $\eta=-\ln \tan (\vartheta / 2)$ and $\vartheta$ is the angle between the muon and the beam direction). In this region the flux of

Manuscript received October 12, 2006; revised February 21, 2007.

D. Primor is with the School of Electrical Engineering, Tel Aviv University, Tel Aviv 69978, Israel and also with CERN, 1211 Geneva 23, Switzerland (e-mail: david.primor@cern.ch).

G. Mikenberg is with the Weizmann Institute, Rehovot 76100, Israel and also with CERN, 1211 Geneva 23, Switzerland.

H. Messer is with the School of Electrical Engineering, Tel Aviv University, Tel Aviv 69978, Israel.

Color versions of one or more of the figures in this paper are available online at http://ieeexplore.ieee.org.

Digital Object Identifier 10.1109/TNS.2007.894817 photons and neutrons can reach $2800 \mathrm{~Hz} / \mathrm{cm}^{2}$ which will cause a significant number of uninteresting contamination hits to appear close to the muon tracks.

Many particle-tracking algorithms have been developed over the years by the High Energy Physics (HEP) community (see for example [2]). Nevertheless, the requirement of high accuracy in an environment with such a high background level is still very demanding. The commonly used technique [1], which first estimates all particle hit locations and then identifies the tracks, becomes complicated in a high background environment in which the number of hits is large. This publication discusses the potential benefit of the detect-before-estimate approach that uses the physical measurements as the input for the track detection process without prior estimating of the hit locations. Another two novel ideas used in this publication are the use of additional background information for reducing the fake track rate, and the use of cluster classification for better track parameter estimation. The rest of the publication is organized as follows: In Section II the CSC detector is described as well as the experimental set up used to evaluate the tracking algorithm. In Section III the track finding approach is described. Section IV presents the Hough transform (HT) as the main tool to provide track identification. The theoretical detection problem is presented in Section $\mathrm{V}$ and the detection performance for the model suggested is calculated. In Section VI the track fitting technique is described, including the cluster classification procedure. The conclusions are presented in Section VII.

\section{The CSC Detector AND the Test BeAm}

\section{A. The ATLAS CSC}

The ATLAS CSC detectors [1] consist of four-layer chambers that give a position measurement based on charge-interpolation. When an energetic particle, presumably a muon, passes through the chamber, it ionizes a local region of the gas that fills the chamber. The ionized cluster of electrons drifts towards a nearby anode wire and a charge avalanche is established. The charge avalanche induces charge on two sets of cathode strips that are mutually perpendicular (there are 192 strips oriented orthogonal to the anode wires and 48 strips parallel to it). The induced charge is spread out over several adjacent strips; each strip receiving a fraction of the total induced charge. The spread of strips that receive charge is called a hit-cluster. The concept is that with the knowledge of the interpolated total charge passing through a layer, calculating the relative magnitudes of both the charge on each strip and the position of the strip in the hit-cluster, one obtains enough information to find the centroid of the charge. The centroid is the point in the chamber where the ionization cluster originated, thus, the position of the energetic 


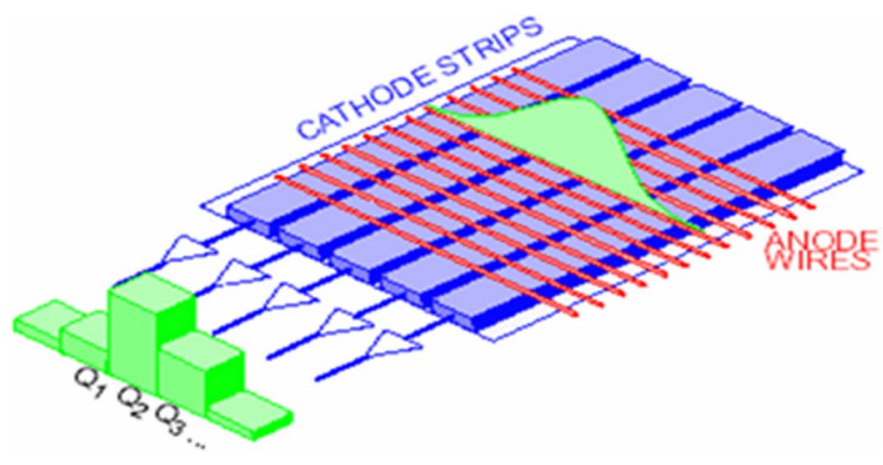

Fig. 1. Charge distribution over the precision strips [1].

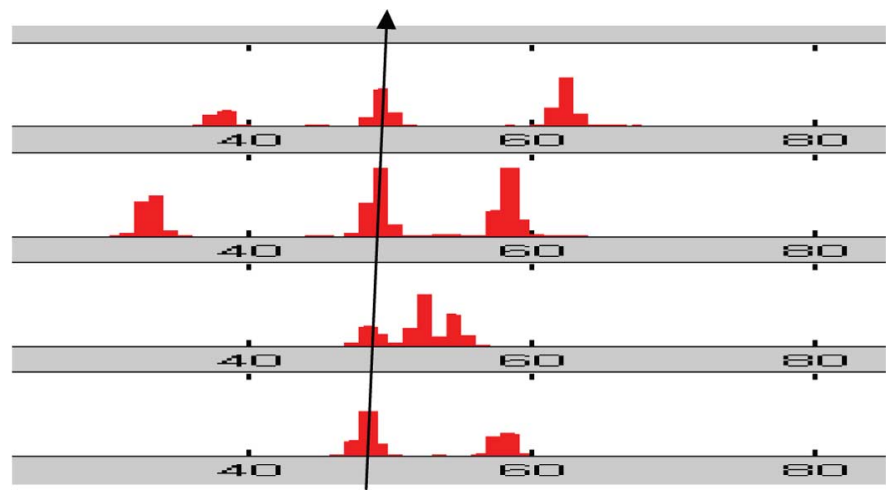

Fig. 2. Hit clusters of a four-layer CSC detector. The axes are the strip number and the CSC layers. Only the hit clusters on the upright arrow belong to the muon track. The other hit clusters are probably due to other particles.

particle's track. Fig. 1 describes the charge induction over the precision strips.

The strip output signal has a bipolar shape in time, where its maximum determines the induced charge over the strip, and its corresponded time (maximum time) is related to the electron clusters drift time. The maximum drift time of about $30 \mathrm{~ns}$ [1], together with a time reference, defines a muon time window. That is the time frame in which a muon is expected to cross the chamber.

The CSC is located in a high radiation area, where the detected fluxes of photons and neutrons can reach $2800 \mathrm{~Hz} / \mathrm{cm}^{2}$. This can lead to a situation where uninteresting particle hits are close to the muon track. The creation of secondary particles $(\gamma$ rays) caused by the interaction of muons with the detector material, the inefficiency of strip channels, and other electronic phenomena such as overflow and crosstalk, can produce false track candidates and reduce the detection performance for the real muon track. Fig. 2 illustrates the hit clusters induced over the four-layer CSC detector. Only those which are on the straight arrow belong to the given muon track.

\section{B. The Test Beam}

The background environment of the LHC experiments will be high, particularly in the forward regions. The expected background rate for the CSC, calculated in [3] is shown in Fig. 3. In order to study the performance in the presence of high background, a CSC chamber was tested at the Gamma Irradiation

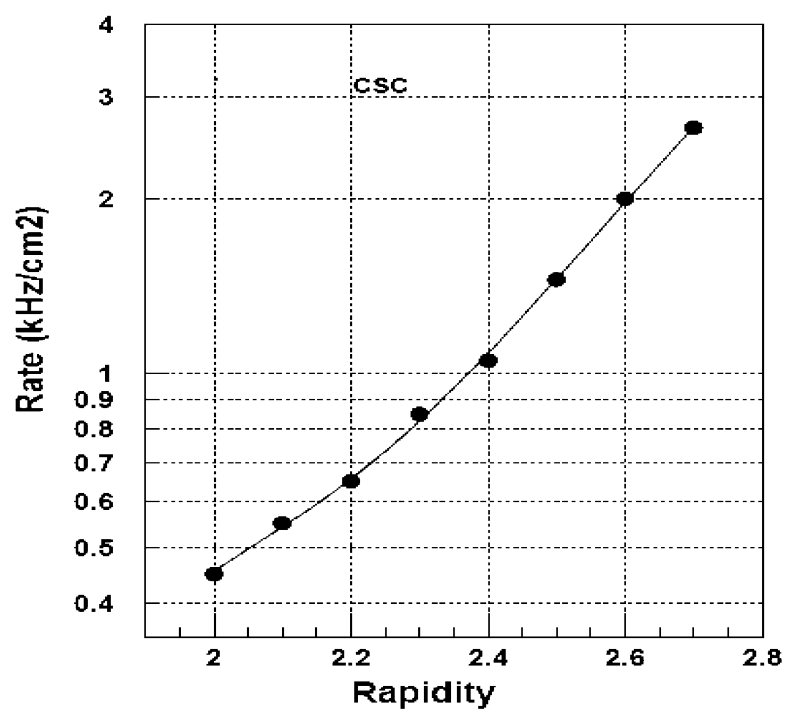

Fig. 3. Expected background rate for the CSC detector [3].

TABLE I

DETECTED BACKGROUND RADIATION RATE IN KHZ/CM ${ }^{2}$ FOR THE 4 CSC LAYERS, AND FOR DIFFERENT LEAD ABSORBER VALUE. THE R02 VALUE CORRESPONDS TO THE HIGHEST INTENSITY, WHILE THE VALUE OF R10 CORRESPONDS TO THE LOWEST

\begin{tabular}{|c|c|c|c|}
\hline Layer & R10 & R05 & R02 \\
\hline $\mathbf{0}$ & 0.717 & 1.412 & 3.238 \\
\hline $\mathbf{1}$ & 0.774 & 1.431 & 3.224 \\
\hline $\mathbf{2}$ & 0.656 & 1.244 & 2.860 \\
\hline $\mathbf{3}$ & 0.595 & 1.185 & 2.562 \\
\hline
\end{tabular}

Facility (GIF) at CERN during July 2004. This facility provided a high energy muon beam in conjunction with a variable high intensity radioactive gamma source $664 G B q^{137} \mathrm{Cs}$. Variable lead absorbers were used to change the radiation intensity. The beam illuminated area of the chambers was determined by trigger counters. The radiation background rate, as observed in the GIF test beam, is summarized in Table I [4].

A detailed description of the summer 1998 test beam setup for the CSC prototype is described in [3]. The main difference between that test and the more recent one is the lack of a Si telescope in the second experiment. The data taken from the recent test beam was used for the analysis described in this publication.

\section{The CSC Performance in High Rate Radiation}

The major reason for the CSC performance degradation at high rates is the overlapping of the cathode strip signals. Since the position in the CSC is obtained by interpolating the induced charge from several cathode strips, even minor disturbances of the signal in one of the strips can disturb the measured position significantly. The anode-cathode crosstalk is another factor that affects the CSC performance. In the CSC, anodes and cathodes lay perpendicular to each other and due to the finite capacitance between them some fraction of anode signal is sensed at the cathode strips. The background at the LHC will be composed mainly of gamma and neutron interactions in the gas and wall materials of the CSC, which can lead to large energy deposits in the chamber. In this case the cross talk signal may be 
large enough to dislocate the track position in the chamber. Another reason for CSC performance degradation studied in the test beam is the crosstalk between adjacent layers. Strips with high charge value will be followed, in many cases, by strips with low charge values in the adjacent layer.

A naïve track reconstruction might try to find all tracks with at least $\mathrm{n}$ collinear hits, where $\mathrm{n}$ is a predefined number, smaller or equal to the number of layers in the chamber. The collinear hit detection probability is estimated by calculating the single layer efficiency. A single layer efficiency is defined as the fraction of events with cluster position inside a fixed range of $\pm 0.3 \mathrm{~mm}$ from the real track. For a background rate of $2.3 \mathrm{KHz} / \mathrm{cm}^{2}$ a single layer efficiency of $75.5 \%$ was found in [3]. Similar results were measured in this experiment.

If the single layer efficiency is $80 \%$, for example, the probability of detecting collinear hits through all the layers is 0.41 , according to:

$$
P_{D}(f)=\sum_{i=n}^{4}\left(\begin{array}{l}
4 \\
i
\end{array}\right) \cdot f^{i} \cdot(1-f)^{4-i}
$$

where $f$ is the single layer efficiency and $n=4$. The use of at least 3 collinear hits, results in probability of detection of 0.82 . In order to get a higher probability of detection, one should use $n=2$. In this case $P_{D}(f)=0.97$ is achieved, but the track fake rate increases dramatically, as described in the next sections.

This publication introduces a new track-finding approach taking into account hits that are not necessarily collinear with the others, probably due to a nearby background particle. Since a muon induces charge on each layer it crosses, there should be an indication of the muon or of a background particle that masked the muon in each layer. The new approach uses the collinear hits together with the indication of background particles in order to achieve high probability of detection with low fake track rate. It is shown in the next chapters that the new approach achieves probability of detection which approaches $100 \%$, while reducing the track fake rate significantly.

\section{The PROPOSED APPROACH}

The suggested muon detection approach is based on 3 ideas discussed throughout this publication: (a) the detect-before-estimate approach, (b) muon track detection using additional information, and (c) muon track parameter estimation using selective clusters.

\section{A. The Detect-Before-Estimate Procedure}

In the presence of a noisy background, the number of recorded hits is much larger than the number of the muon hits. In the background environment of the LHC, one can expect to get a number of hits, which is about 10 times the number of the muon hits of interest. The efficiency of the CSC detection is, however, close to $100 \%$ in each layer for the detection of a Minimum Ionizing Particle (MIP) such as a muon. It is therefore suggested to use a procedure of detect-before-estimate that detects the muon tracks (four aligned hit clusters) without applying sophisticated calculations to find the hit clusters properties. These calculations should be applied, as a second phase, only for the clusters that are clearly associated to a muon track. This approach aims to reduce the overall algorithmic complexity in a noisy environment.

\section{B. Low Fake Rate Muon Track Detection}

The major reason for layer inefficiency at high background rates is the overlapping of background strip signals, mainly photons and neutrons, with muon strip signals. Since the hit time of photon and neutron background is uncorrelated with the muon hit time, it is usually possible to distinguish between a potential muon hit and a background hit. A muon that crosses the CSC layers produces a hit cluster at the interaction point with each layer (hit point). These hit clusters contain strips with signal peak time (maximum time) inside the muon time window. These strips are defined as potential strips. At high background rates, these clusters may overlap with hit clusters originated by background particles, producing "dirty" clusters. In many cases, these dirty clusters contain strips with maximum time outside the muon time window. These strips are defined as mask strips. A track candidate must have either a potential strip or a mask strip in each layer. Thus, the closest strip to the muon hit point at each layer should be either a potential strip or a mask strip. A CSC track detection algorithm that uses the potential strips, as well as the mask strips, is proposed. It will be shown that this approach reduces the fake track rate significantly without reducing the detection probability.

\section{Muon Track Parameter Estimation Using Selective Clusters}

In order to estimate the track parameters, the muon hit positions should be estimated at the different layers. Even a minor disturbance of the signal on at least one of the cluster strips may disturb the measured position. A classification of the hit clusters into "clean" and "dirty" clusters is suggested. The "clean" clusters are those that are well separated from other clusters and have the ideal charge distribution according to the Matheison distribution [5]. The "dirty" clusters are either close to other clusters, have mask strips, or have a spatial charge different from the Matheison distribution. The hit positions are estimated only for the "clean" clusters, and are used for estimating the track parameters.

\section{The Hough Transform FOR Track Finding}

\section{A. The Conventional Hough Transform}

The problem of track segment identification in detector data is comparable to the problem of line identification in a noisy image [2], a problem that occurs often in image analysis. One solution is related to the HT [6]-[8]. Points in the image are transformed into lines in a line parameter space. Lines in the parameter space corresponding to collinear points that cross each other at one point. This point defines the spatial parameters of the line through the collinear points.

In practice, the parameter space is divided into an array of discrete cells. When a point in the image space is transformed into a line in the parameter space, all cells crossed by the line are incremented. If $n$ points are approximately collinear, the line parameters in the image space correspond to a local maximum in 


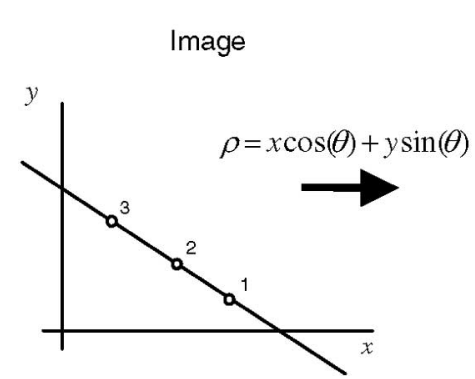

Line parameter space

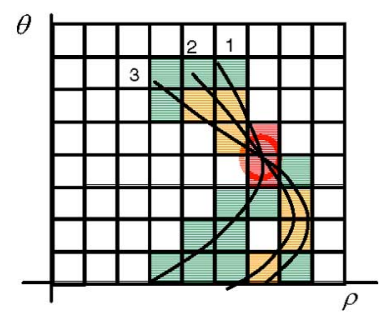

Fig. 4. Points in the image (left) are transformed into lines in a line parameter space (right). Lines in the parameter space corresponding to collinear points will cross each other at one point.

the parameter space. The local maximum is produced by the approximate intersection of $n$ lines. Thus, the Hough transform reduces the problem of searching for collinear points in the image to that of looking for cells in the parameter space, which are local maxima. Fig. 4 illustrates the transform of three collinear points in an image to the Hough parameter space.

The performance of the HT-based algorithm has been analyzed and compared to other detection methods in the presence of additive noise [9], [10]. It was shown that in the presence of Gaussian, uniform or Laplacian noise, both Hough transforms and Gaussian-based optimal algorithms have good detection performance [11]. While the Gaussian-based optimal algorithm has superior location estimation performance, it is computationally more intensive. Furthermore, due to the presence of interference, the Gaussian noise assumption cannot always be applied for particle detectors, and a more robust detection method is needed. The HT algorithm was shown to be a very robust approach when compared to other algorithms [12].

\section{B. The Modified Hough Transform}

Using the specific characteristics of the CSC muon detector, it is possible to modify the standard Hough transform and reduce the algorithm complexity. The fact that one should look for straight lines within the detector layers starting at the first layer and ending in the last (fourth) layer can simplify the calculation. Instead of using the parametric form $y=k x+d$ or the polar form $\rho=x \cos \theta+y \sin \theta$, a two- point form is used:

$$
y-y_{1}=\frac{y_{2}-y_{1}}{x_{2}-x_{1}}\left(x-x_{1}\right) .
$$

This form represents a line that is going through two points: $\left(x_{1}, y_{1}\right)$ and $\left(x_{2}, y_{2}\right)$. For the CSC detector, $y_{1}, y_{2}$ are both known (the layer locations), so it is possible to implement a transform from any point $(x, y)$ in the track to a straight line in the parameter space using $\left(x_{1}, x_{2}\right)$ as the only two unknown parameters. This formulation enables one to put constraints on the tracks easily. For example, if $x_{1}$ and $x_{2}$ are limited to the maximum strip number, only lines that go through the first and the fourth layer are represented in the Hough space. Additional simplification can be obtained by applying an angular constraint, i.e., the transform will be applied only for lines roughly projected to the interaction point (where the proton beams collide).
TABLE II

The Track Finding EFficiency and FaKe Track Rate For DIFFERENT THRESHOLD VALUES FOR R02 FILES

\begin{tabular}{|c|c|c|c|c|}
\hline$n_{p}$ & $\begin{array}{c}\text { Hough } \\
\text { efficiency } \\
\text { using the } \\
\text { mask strips }\end{array}$ & $\begin{array}{l}\text { Hough } \\
\text { efficiency } \\
\text { without the } \\
\text { mask strips }\end{array}$ & $\begin{array}{l}\text { Hough } \\
\text { fake tracks } \\
\text { using the } \\
\text { mask strips }\end{array}$ & $\begin{array}{l}\text { Hough fake } \\
\text { tracks } \\
\text { without the } \\
\text { mask strips }\end{array}$ \\
\hline 4 & 0.957 & 0.957 & $\mathbf{0 . 3}$ & 0.3 \\
\hline 3 & 0.996 & 0.996 & $\mathbf{0 . 3}$ & 0.5 \\
\hline 2 & 0.999 & 0.999 & $\mathbf{0 . 3 1}$ & 6.7 \\
\hline
\end{tabular}

Each cell in the Hough parameter space has three values. The first one is the number of potential strips with transforms crossing the cell. The second value is the number of mask strips with transforms crossing the cell. The third one is the sum of the strip charges associated with the cell. The first and second values are used for finding the local maximum above a predefined threshold. The third value is used for filtering the parameter space by selecting the lines with the maximum charge.

\section{Hough Transform Results for the Test Beam Data}

In order to check the detection performance of the Hough transform, the algorithm results should be compared to the real tracks ("truth"). Since in the discussed test beam there was no other measurement to cross check the information collected with the CSC, the true information was not available.

In order to overcome this problem, the data received when the gamma source was not activated (clean data) was used for generating a "truth" reference. Only clean data events with 4 hits, one from each layer of the CSC, were taken as the "truth". Then, the position of each cluster was calculated using the ratio algorithm [3] and a linear regression was applied for finding the track. This track is considered as the "truth".

The generated "truth" was combined with data taken when the source was activated (noisy data). The track efficiency was defined as the fraction of tracks that were found in a distance of $1 \mathrm{~mm}$ from the "truth". The fake tracks were defined as the total number of tracks found, minus the two real tracks (one from the clean data file, and the other from the noisy data file).

Table II describes the results of the track-finding algorithm using the R02 file for different cell thresholds. The threshold is the combination of minimum number of potential strips $n_{p}$ and the requirement of complementary mask strips at the layers where no potential strips were found. It can be seen that the use of the mask strips reduces the number of reconstructed fake tracks significantly.

Figs. 5 and 6 describe the track finding efficiency and fake track rate for $n_{p}=3$ and requiring complementary mask strips for different background levels.

\section{Detection Analysis of the Hough Transform}

Performance evaluation of the Hough transform based algorithm is done using a comparison between the algorithm experimental results and the theoretical detection results. The theoretical detection performance is calculated using the following model assumptions:

a) A muon track produces muon hits in all layers.

b) A muon hit has a spatial Matheison distribution [5], and its maximum charge follows the Landau distribution [13]. 


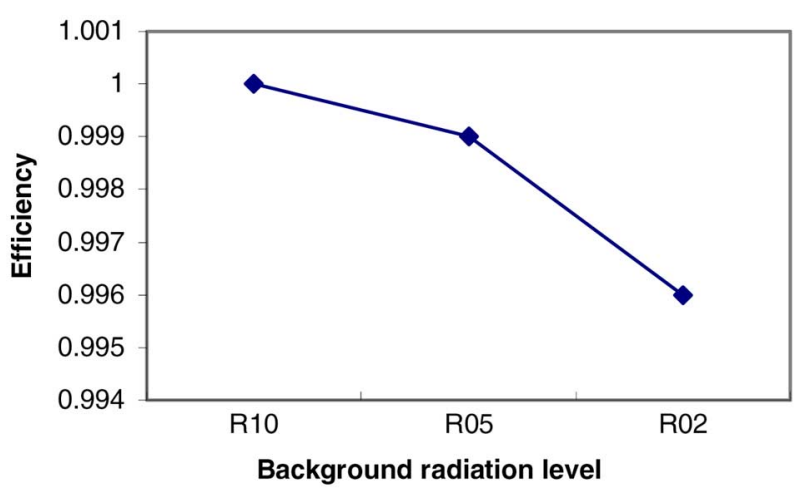

Fig. 5. Track finding efficiency using the Hough transform for the different background level files.

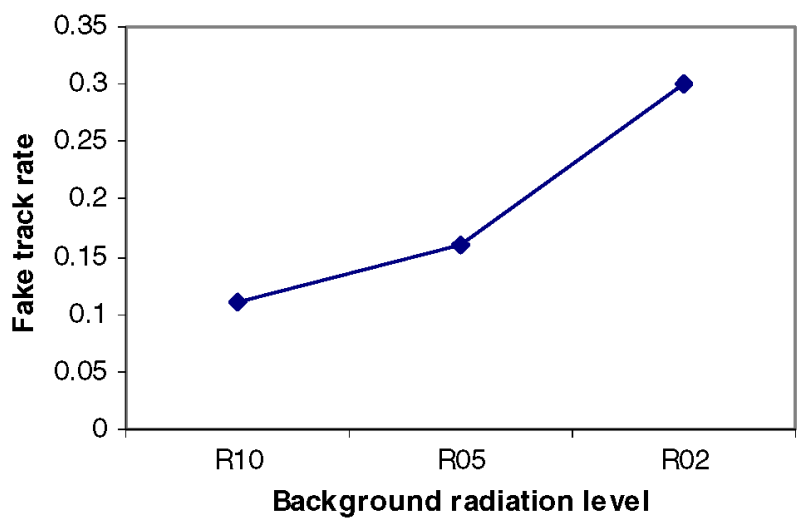

Fig. 6. Fake track rate values of the Hough transform for the different background level files.

c) The electronic noise is independent Gaussian adaptive noise.

d) The background hits are mutually uncorrelated.

e) The background hits can be either muon-like hits (produce clusters with potential strips) or mask hits (produce clusters with mask strips). The probabilities of both cases are taken from the test beam experimental probabilities.

f) A background hit has a spatial Matheison distribution (it is assumed that it crosses the chamber perpendicularly), and its charge is always above the threshold.

g) A background hit in one layer might cause a second hit in the adjacent layer, due to electronic cross talk between the layers.

\section{A. Performance Analysis of Hit Detection}

Define a matrix $\mathbf{Y}$ :

$$
\mathbf{Y}_{i j}=y_{i}(j), j=1 \ldots M, i=0 \ldots L
$$

where $y_{i}(j)$ is the charge of the $\mathrm{j}$-th strip in the $\mathrm{i}$-th layer, $M$ is the number of strips in a layer, and $L$ is the number of layers. The matrix $\mathbf{Y}$ is a multivalued array of dimension $M \times L$ (in this case $M=192, L=4)$. The transformation of the multivalued array $\mathbf{Y}$ to a binary array requires activity detection for each strip in the detector. Practically, a strip threshold $\gamma$ is used, and strips with maximum charge above the threshold are marked as
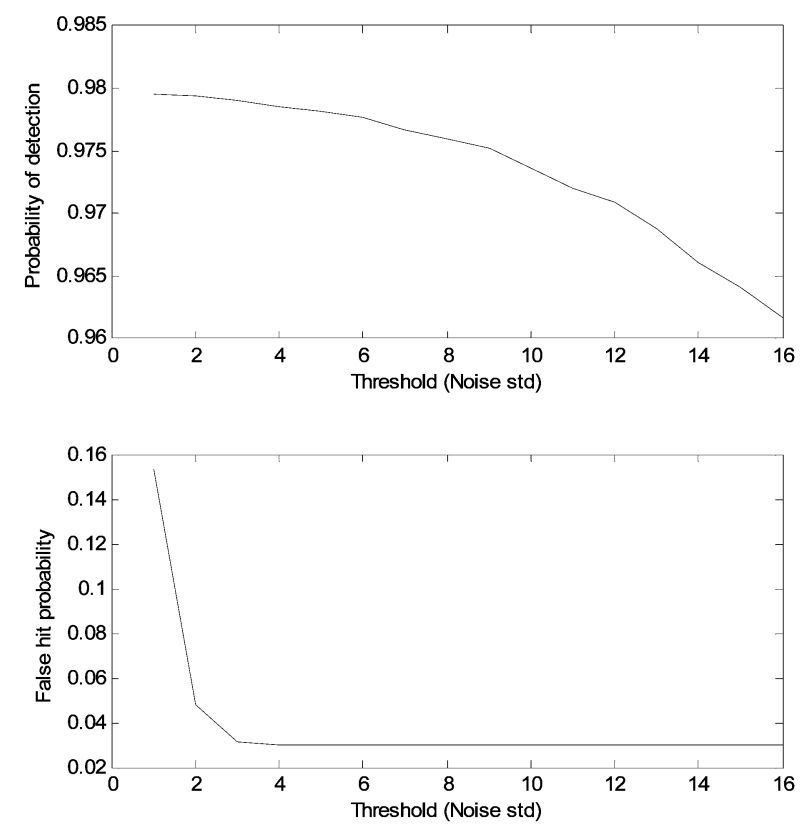

Fig. 7. The probability of detection (top) and of false hit (bottom) for a single strip vs. the threshold of the activity detection, $\gamma$. The noise standard deviation (std) was calculated from the test beam data.

active strips. The probability of detecting a muon hit in a layer $\mathrm{i}$ and strip $\mathrm{j}$ is given by:

$$
p_{D}\left(\gamma, p_{\bar{a}}\right)=p_{m}\left(\mathbf{Y}_{i j}>\gamma \mid H_{1}, \bar{a}\right) \cdot p_{\bar{a}}
$$

where $\mathbf{Y}_{i j}$ is the charge read, and $H_{1}$ is the hypothesis that the strip was hit by a muon. $p_{\bar{a}}$ is the probability that no photon or other particle masks the muon signal. The probability $p_{m}\left(\mathbf{Y}_{i j}>\gamma \mid H_{1}, \bar{a}\right)$ is the probability that the strip maximum amplitude is above the threshold $\gamma$, given that the strip was hit by a muon and there was no photon that masked the muon signal. It is assumed that the electronic noise level is the same for all the strips, and the probability of a background particle hit does not depend on the strip position. Thus, the probability $p_{D}\left(\gamma, p_{\bar{a}}\right)$ is the same for all the strips. In Appendix $\mathbf{A}$ it is shown how to derive $p_{D}\left(\gamma, p_{\bar{a}}\right)$ for a given $p_{\bar{a}}$.

There are two main sources of false hits for the test beam data; the additive electronic noise, and the photon background. The probability of a false hit is the probability that one or more events happened (or one minus the probability that none of them happened).The false hit probability for layer $\mathrm{i}$ and strip $\mathrm{j}$ is:

$$
p_{F}\left(\gamma, p_{\bar{b}}\right)=1-p_{m}\left(\mathbf{Y}_{i j}<\gamma \mid H_{0}\right) \cdot p_{\bar{b}}
$$

where $p_{m}\left(\mathbf{Y}_{i j}<\gamma \mid H_{0}\right)$ is the probability that the strip maximum amplitude is under the threshold given there was no muon. $p_{\bar{b}}$ is the probability that there is no muon-like hit caused by a photon. In Appendix B it is shown how to derive $p_{F}\left(\gamma, p_{\bar{b}}\right)$ for a given $p_{\bar{b}}$.

Fig. 7 shows the theoretical probabilities of hit detection and of false hit detection for a single strip as a function of the strip threshold and for $p_{\bar{b}}=97 \%$. It can be seen that for $\gamma>3$ the 
false hit probability does not depend on $\gamma$ and is equal to 0.3 . The false hit probability in this case is caused only by photons.

\section{B. Performance Analysis of Line Detection}

The line detection includes executing the modified Hough transform on a binary array. A cell threshold $\Gamma$ is used in each cell of the parameter space in order to detect a line. The probability of the line detection depends on the probability of detecting the hits in the previous phase, and on the cell threshold $\Gamma$. The cell threshold $\Gamma$ is the minimum number of potential strips. Since the efficiency of the CSC detection is close to $100 \%$ in each layer, the sum of the potential pixels and mask pixels should be 4 .

Since the maximum charge distribution for each layer follows the Landau distribution and the noise in the different layers is uncorrelated, it is reasonable to assume that the events of getting muon hits at different layers are independent.

Therefore, the probability of detecting a line is the probability of detecting at least $\Gamma$ collinear points:

$$
\begin{aligned}
p_{\mathrm{Dl}}\left(\gamma, \Gamma, p_{\bar{a}}\right) & =p\left(A_{c}>\Gamma \mid H_{1}\right) \\
& =\sum_{i=\Gamma}^{4}\left(\begin{array}{c}
i \\
4
\end{array}\right) \cdot p_{D}^{i}\left(\gamma, p_{\bar{a}}\right)\left(1-p_{D}\left(\gamma, p_{\bar{a}}\right)\right)^{4-i}
\end{aligned}
$$

where $A_{c}$ is the accumulator output for the Hough cell and $p_{D}\left(\gamma, p_{\bar{a}}\right)$ is defined in (4).

The probability of detecting a false line depends on the probability of detecting false hits in the previous phase, on the cell threshold $\Gamma$, and on non-linear affects between the different layers (such as cross talk). The analytical expression for a false line probability is derived in Appendix C.

Fig. 8 describes the probability of line detection and the probability of false line detection for the analytical calculation and the experimental results. It can be seen that a very high probability of line detection is achieved using the proposed method, while keeping the probability of false line low. Note, however, that while the theory predicts well the detection probability, it does not perform as well for the probability of false line detection. The probability of false line detection is very close to the theoretical result for $\gamma>10$. For $\gamma<10$, the theoretical result is further from the experimental results. A possible explanation is that other non-linear effects were not taken into account in the model (such as anode-cathode crosstalk), and that there may be degradation in detection performance due to the quantization of the parameter space.

\section{The Track FitTing}

In order to derive the track parameters, one should estimate the muon hit positions at the different layers. For each track candidate, the group of strips above the threshold which are close to the track are taken as hit clusters. Each cluster is classified into "clean" and "dirty" cluster. A "clean" cluster is a cluster which has an ideal charge distribution according to the Matheison distribution [5], all its strips are potential strips, and is well separated from other clusters. A "dirty" cluster is the one which does not follow at least one of the requirements described for the "clean" clusters. For example, for the test beam data with
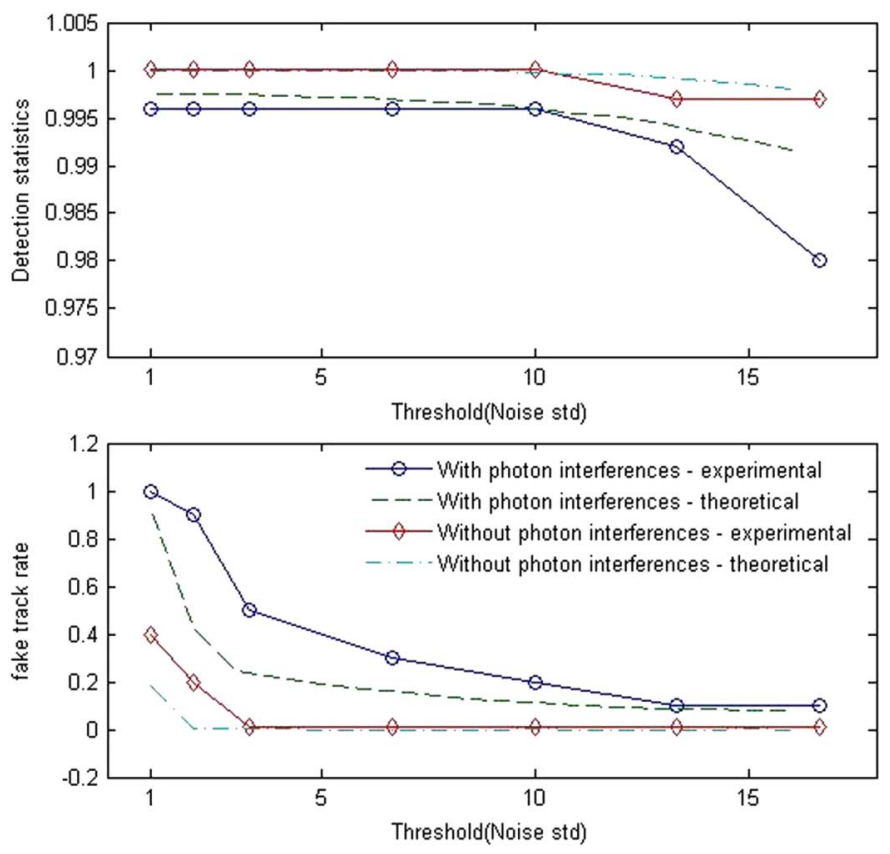

Fig. 8. Experimental and theoretical detection results vs. the strip threshold $\gamma$, for two cases: (a) no interfering photon source and (b) existence of an interfering photon source. For both cases the Hough cell threshold is set to 3 . The upper graph describes the experimental statistics of the line detection success and the theoretical probability of line detection. The lower graph describes the experimental statistics of the false line detection and the theoretical probability for false lines.

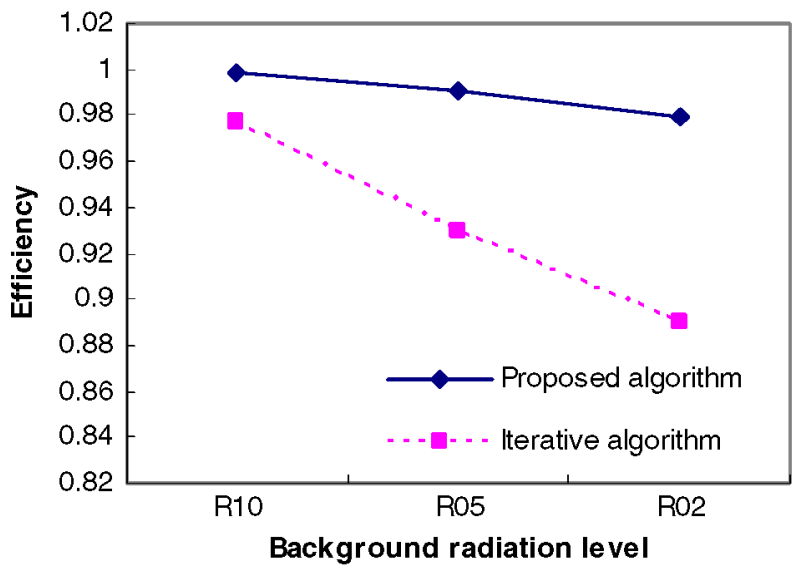

Fig. 9. Track fitting efficiency of the proposed algorithm and of the iterative algorithm taken from [3], for different background sources.

the highest background rate (R02) about one fifth of the clusters due to muons are classified as "dirty" clusters, and about one tenth of the clusters due to background particles are classified as "clean" clusters. The ratio algorithm described in [3] is used for calculating the hit position only for the "clear" clusters. Then, these hit points are taken as the input for the linear regression process.

The track finding efficiency is defined as the fraction of tracks that were found in a distance of $0.5 \mathrm{~mm}$ from the generated "truth". In Fig. 9 the track fitting efficiency of the proposed method is compared to the iterative algorithm suggested in [3]. In [3] a linear regression is applied for all hits as a first step. Then, the hit with the biggest residual is omitted, and the linear 
regression is applied again. Since we have only 4 layers, the regression procedure can be applied a maximum of 3 times. It can be seen in Fig. 9 that the proposed approach has a better track fitting efficiency of about $8 \%$ for the highest background rates.

\section{CONCLUSION}

Local track identification in a high-background environment is one of many new challenges facing the detectors of the LHC. A new algorithm based on several novel ideas is introduced. The detect-before-estimate approach, which aims to reduce the complexity in the presence of high radiation background, is described. A modification of the Hough transform is used for the track finding procedure. It is a multilayer Hough transform that uses additional information for identifying possible mask particles, and exploits the inherent high efficiency of the CSC detector. It is shown that, at high background rates, use of the mask strips can reduce the track fake rate by about $50 \%$. Moreover, the track finding experimental results are very close to the analytical calculation. Since even minor disturbances of the signal on at least one of the strips may disturb the measured position, the classification of the cluster into "clean" and "dirty" is very useful. It is shown that using exclusively "clean" clusters in the track fitting procedure, results in a better track parameter estimation. A comparison between the proposed algorithm and the iterative linear regression algorithm [3] shows a significant advantage for the former.

\section{APPENDIX A}

\section{PROBABILITY OF Hit DETECTION}

It is assumed that the strip maximum charge distribution follows the Landau distribution which is approximated by [13]:

$$
P(s)=\sqrt{\frac{1}{2 \pi} \exp \left(-\frac{1}{2}\left(s+e^{-s}\right)\right)}
$$

Then, the probability that the strip maximum amplitude is above the threshold $\gamma$ is:

$$
\begin{aligned}
& p_{m}\left(\mathbf{Y}_{i, j}>\gamma \mid H_{1}, \bar{a}\right)=\int_{\gamma}^{\infty} p_{m}\left(y \mid H_{1}, \bar{a}\right) d y \\
&=\int_{\gamma}^{\infty} \int_{-\infty}^{\infty} p\left(y \mid H_{1}, \bar{a}, s\right) p\left(s \mid H_{1}, \bar{a}\right) d s d y \\
&=\int_{\gamma}^{\infty} \int_{-\infty}^{\infty} \frac{1}{\sqrt{2 \pi} \sigma} \exp \left(\frac{(y-s)^{2}}{2 \sigma^{2}}\right) \\
& \cdot \frac{1}{\sqrt{2 \pi}} \exp \left(-\frac{1}{2}\left(s+e^{-S}\right)\right) d s d y \\
&= \int_{\gamma}^{\infty} \int_{-\infty}^{\infty} \frac{1}{2 \pi \sigma} \\
& \times \exp \left(\frac{(y-s)^{2}}{2 \sigma^{2}}-\frac{1}{2}\left(s+e^{-s}\right)\right) d s d y \hat{=} L G(\gamma) .
\end{aligned}
$$

Thus, using (4), the probability of detecting a muon hit in a certain strip is:

$$
p_{D}\left(\gamma, p_{\bar{a}}\right)=L G(\gamma) \cdot p_{\bar{a}}
$$

Since the photon usually has amplitude higher than that of the muon, it is assumed that every photon hit before the muon time window could potentially mask a muon. It was verified using the data from a test beam that the photon background for data file R02 masks the muon hits in about $2 \%$ of the cases $\left(p_{\bar{a}}=98 \%\right)$

\section{APPENDIX B \\ PROBABILITY OF FALSE Hit}

It is assumed that the electronic noise is an additive white Gaussian noise $(\mathrm{AWGN}) \sim N\left(0, \sigma^{2}\right)$. Thus, the probability that the electronic noise is bellow the threshold is given by:

$$
p_{m}\left(\mathbf{Y}_{i j}<\gamma \mid H_{0}\right)=1-Q\left(\frac{\gamma}{\sigma}\right)
$$

where $Q(x)=\int_{x}^{\infty}\left(1 / \sqrt{2 \pi} \exp \left(-(1 / 2) t^{2}\right) d t\right)$

It was found in the CSC test beam data that $p_{\bar{b}}=97 \%$. Therefore, for this experiment, using (5) the false hit is:

$$
\begin{aligned}
& p_{F}\left(\gamma, p_{\bar{b}}\right) \\
& \quad=1-p_{m}\left(\mathbf{Y}_{i j}<\gamma \mid H_{0}\right) \cdot p_{\bar{b}} \\
& \quad=1-\left(1-Q\left(\frac{\gamma}{\sigma}\right)\right) \cdot 0.97 \\
& \quad=0.97 \cdot Q\left(\frac{\gamma}{\sigma}\right)+0.03 .
\end{aligned}
$$

\section{APPENDIX C \\ Probability of a FALSE Line Detection}

There are four physical sources for a false hit; white electronic noise, photon background, delta electrons and other non linear effects such as cross talk and overflow. Though the electronic white noise is layer independent, there is a dependency between the layers for a delta electron hit and a photon hit. A delta electron can cause more than one hit per layer and might be seen like a muon. A photon with high charge can create a second hit in the adjacent layer due to the electronic cross talk between the layers. Using the test beam data, it was verified that the probability of having a false line due to a delta electron that creates hits in more than one layer is very small. Therefore this case is neglected in this analysis. The probability of a false line is defined as the probability of having one or more false lines per event.

The approach for calculating the false line probability is:

a) For each $0<n<N_{s}$ find the probability of having $\mathrm{n}$ false hits in a certain layer where $N_{s}=192$ is the number of strips in the layer. This probability is defined as $p_{F}^{(n)}(\gamma)$.

b) Find the probability of having at least one detectable line that is collinear with one of the false hits given 
from the previous stage. This probability is defined as $p_{\text {Fcol }}(\gamma, \Gamma \mid n)$.

Then, the probability of a false line is the probability of one or more false hits in a certain layer and the combination of one or more collinear hits in the other layers:

$$
p_{\mathrm{Fl}}(\gamma, \Gamma)=\sum_{n=1}^{192} p_{\mathrm{Fcol}}(\gamma, \Gamma \mid n) \cdot p_{F}^{(n)}(\gamma) .
$$

The probability of having $\mathrm{n}$ false hits in a certain layer is composed of the probability of having $n_{1}$ false hits due to electron noise $\left(p_{\mathrm{Fe}}^{\left(n_{1}\right)}(\gamma)\right)$ and $n-n_{1}$ false hits due to photons hits $\left(p_{\mathrm{Fp}}^{\left(n-n_{1}\right)}\right)$. Then:

$$
p_{F}^{(n)}(\gamma)=\sum_{j=1}^{n} p_{\mathrm{Fp}}^{(j)} \cdot p_{\mathrm{Fe}}^{(n-j)}(\gamma)
$$

where $p_{\mathrm{Fe}}^{(m)}(\gamma)$ is the probability to have any $m$ false hits due to the electronic noise in a certain layer:

$$
p_{\mathrm{Fe}}^{(m)}(\gamma)=\left(\begin{array}{c}
m \\
N_{s}
\end{array}\right) p_{\mathrm{Fe}}^{m}(\gamma)\left(1-p_{\mathrm{Fe}}(\gamma)\right)^{N_{s}-m}
$$

and $p_{\mathrm{Fe}}$ is the strip probability for false hit due to the electronic noise.

In the same way, $p_{\mathrm{Fp}}^{(m)}$ is the probability to have any $m$ false hits due to the photons in a certain layer:

$$
p_{\mathrm{Fp}}^{(m)}=\left(\begin{array}{c}
m \\
N_{s}
\end{array}\right) p_{\mathrm{Fp}}^{m}\left(1-p_{\mathrm{Fp}}\right)^{N_{s}-m}
$$

where $p_{\mathrm{Fp}}$ is the strip probability of a false hit due to the photon.

The probability of having a collinear line with a given false hit is:

$$
p_{\mathrm{col} 11}(\gamma, \Gamma)=\sum_{j=\Gamma-1}^{4}\left(\begin{array}{l}
j \\
4
\end{array}\right) \cdot p_{F}\left(\gamma, p_{\bar{b}}\right)^{j}\left(1-p_{F}\left(\gamma, p_{\bar{b}}\right)\right)^{4-j}
$$

where $p_{F}\left(\gamma, p_{\bar{b}}\right)$ is defined in (5). The probability of having at least one collinear line for $\mathrm{n}$ possibilities ( $\mathrm{n}$ false hits in a certain layer) is:

$$
p_{\text {Fcol }}(\gamma, \Gamma \mid n)=1-\left(1-p_{\text {coll }}(\gamma, \Gamma)\right)^{n} .
$$

The cross talk between the layers may produce two false hits for one photon hit. Thus, (C-5) can be approximated by:

$$
p_{\mathrm{col} 1}(\gamma, \Gamma) \approx \sum_{j=\Gamma-2}^{4}\left(\begin{array}{l}
j \\
4
\end{array}\right) \cdot p_{F}\left(\gamma, p_{\bar{b}}\right)^{j}\left(1-p_{F}\left(\gamma, p_{\bar{b}}\right)\right)^{4-j} .
$$

Since the cross talk depends on the amplitude of the photon hit, the probability $p_{\text {col1 } 1}(\gamma, \Gamma)$ is bounded by the two expressions of (C-5) and (C-7).

\section{ACKNOWLEDGMENT}

The authors would like to thank the CSC community, and especially Prof. V. Polychronakos of Brookhaven National $\mathrm{Lab}$ for providing access to the CSC test beam data and for improving their understanding of the CSC detector with his valuable advice. The authors would also like to thank M. Schernau of the University of California for providing an excellent CSC event viewer, improving their understanding of the CSC tracking problem. Finally, the authors would like to thank Prof. E. Etzion of Tel Aviv University and Dr. S. Goldfarb of the University of Michigan for editing this publication and providing useful advice.

\section{REFERENCES}

[1] Atlas Muon Spectrometer Tech. Design Rep., CERN-LHCC-97-22, 1997.

[2] R. Fruhwirth, "Data analysis techniques for high-energy physics," Cambridge Monographs Particle Phys., Nucl. Phys. Cosmology, pp. $110-220,2000$.

[3] A. Gordeev, "CSC performance at high background rates," CERN Geneva, Switzerland, Oct. 19, 1999, ATL-MUON-2000-005.

[4] [Online]. Available: http://positron.ps.uci.edu/ schernau/ROD/SIT/ results/

[5] E. Mathieson and J. S. Gordon, "Cathode charge distributions in multiwire chambers II. Approximate and empirical formulae," Nucl. Instrum. Methods Phys. Res. A, vol. A227, pp. 277-0, 1984.

[6] J. Illingworth and J. Kittler, "A survey of the hough transform," Comput. Vis. Graph. Image Process., vol. 44, no. 10, pp. 87-116, 1988.

[7] R. O. Duda and P. E. Hart, "Use of the hough transform to detect lines and curves in pictures," Commun. Ass. Comput. Mach., vol. 15, no. 11-15, 1972.

[8] P. V.C. Hough, "Method and means for recognizing complex patterns," U.S Patent 3069 654, Dec. 1962.

[9] D. J. Hunt, L. W. Notle, and W. H. Ruedger, "Performance of the hough transform and its relationship to statistical signal detection theory," Comput. Vis. Graph. Image Process., vol. 43, pp. 221-238, 1988.

[10] Z. Hu and H. Leung, "Statistical performance analysis of track initiation techniques," IEEE Trans. Signal Process., vol. 45, no. 2, Feb. 1997.

[11] D. J. Hunt, L. W. Notle, and A. R. Reibman, "Hough transform and signal detection theory performance for images with additive noise," Comput. Vis. Graph. Image Process., vol. 52, pp. 386-401, 1990.

[12] J. Sheinvald and N. Kiriaty, "On the magic of SLIDE," Mach. Vis. Appl., vol. 9, pp. 251-261, 1997.

[13] J. E. Moyal, "Theory of ionization fluctuations," Philos. Mag., vol. 46, pp. 263-0, 1955 . 